\title{
Increased learning in a college physics course with timely use of short multimedia summaries
}

\author{
Spencer Dunleavy $\odot{ }^{1,2}$ Greg Kestin, ${ }^{2}$ Kristina Callaghan, ${ }^{2}$ \\ Logan McCarty $\odot,{ }^{2,3}$ and Louis Deslauriers $\circledast^{2, *}$ \\ ${ }^{1}$ School of Medicine, Columbia University, New York 10032, USA \\ ${ }^{2}$ Department of Physics, Harvard University, Massachusetts 02138, USA \\ ${ }^{3}$ Department of Chemistry and Chemical Biology, Harvard University, Massachusetts 02138, USA
}

(Received 30 August 2021; accepted 10 December 2021; published 28 January 2022)

\begin{abstract}
The typical introductory physics lecture requires students to consolidate and assimilate a large quantity of complex information that is often novel to them. This can leave students overwhelmed, slow the pace of their learning, and lower their motivation. We find that carefully designed multimedia summaries in the form of one-minute videos and short text summaries can significantly increase students' understanding of the material as well as their ability to organize information into a useful mental framework, as measured by their performances on a concept mapping exercise and a conceptual test of learning. Notably, we show that these improvements can be achieved with negligible increase in overall time students spend on the course material each week. We discuss reasons why these short postlecture summaries helped students learn more, namely, that (i) they likely increased students' ability to chunk and organize information while minimizing the extraneous cognitive load imposed by the materials, and (ii) they likely improved students' ability to consolidate and transfer knowledge through the use of contrasting cases. We provide a set of detailed recommendations that instructors can use to develop effective postlecture multimedia summaries. We suggest that one of the most important and impactful recommendations is incorporating student thinking in the design of these types of summaries informed by the input of qualified former students or teaching assistants with significant experience interacting with students in the course.
\end{abstract}

DOI: 10.1103/PhysRevPhysEducRes.18.010110

\section{INTRODUCTION}

In undergraduate introductory science, technology, engineering, and math (STEM) courses, students are required to simultaneously handle a large quantity of highly complex information, often with entirely novel material, at a rate they may not have previously experienced. This combination of quantity and complexity presents a challenge for how STEM instructors strike a balance between covering the full extent of the material in the curriculum without overwhelming students. With the goal of maximizing student learning, instructors often need to develop additional strategies and course materials to overcome these barriers as effectively as possible.

A fundamental part of learning is organizing and chunking information; decades of cognitive science research have demonstrated the importance of breaking

\footnotetext{
*To whom correspondence should be addressed. louis@physics.harvard.edu

Published by the American Physical Society under the terms of the Creative Commons Attribution 4.0 International license. Further distribution of this work must maintain attribution to the author(s) and the published article's title, journal citation, and DOI.
}

complex information into digestible chunks to help learners retain and use the information [1-4]. Cognitive load theory, which posits that learning is inhibited when the learning task itself is too cumbersome [5-8], provides the theoretical underpinnings for these learning strategies. At its essence, the instructional goal is to maximize productive efforts involving germane cognitive load, while minimizing extraneous cognitive load $[6,9,10]$ —in other words, processing of information into mental frameworks to apply in novel scenarios, while minimizing barriers that arise based on the way we instruct learners. The role of a teacher is to not only draw students' attention to important details [11], but to also consider which details should be identified, how they are ordered, in what medium they should be presented, and with what complexity they are described. In particular, an instructor can help students deepen and apply their understanding in new contexts by organizing and chunking important topics and concepts. This may be especially important when students are processing novel information presented in lecture, as the quantity and complexity of information may not yet have been incorporated into useful mental frameworks (i.e., schemas).

Instructors commonly attempt to help students organize and chunk the information presented in lecture by 
providing them with summaries of lecture materials. The effectiveness of this approach has been supported in pedagogical research across disciplines [12-17]. For example, both student-generated and instructor-generated summaries have been shown to improve secondary students' understanding of scientific concepts [12]; however, students using instructor-generated summaries performed better on visualization and transfer of knowledge tests compared to students using their own summaries. This suggests that instructor-produced summary materials may provide a scaffolding that enables students to organize and chunk novel concepts from lecture.

Carefully designed multimedia materials have also been shown effective at helping students cope with the high volume and complexity of information presented in lectures. For example, students exposed to narrated, animated prelecture videos in an introductory electricity and magnetism course significantly improved their understanding of the course material, as measured by their performance on tests of learning [18]. In another study that measured how much students learn from lecture demonstrations in introductory physics courses, online video versions of lecture demonstrations were found to be more effective than their live counterpart-moreover, students' self-reported enjoyment was measured to be the same with both formats [19].

In this paper, we address the question whether short postlecture multimedia summaries can improve students' ability to chunk and organize information into useful schema as well as increase their problem-solving skills. We seek to answer this question through a well-controlled study of postlecture summaries consisting of short videos and texts. We find that carefully designed multimedia summaries can significantly improve students' understanding of the material as well as their ability to create useful mental frameworknotably, we show that these improvements can be achieved with negligible increase in the overall time students spend on the course material each week. These findings are consistent with evidence that chunking $[4,11]$, the use of contrasting cases [20-22], and the perspective of an expert tutor [23-26] can improve student learning.

\section{METHODS}

The study took place in a large enrolment calculus-based introductory physics course at Harvard University. With topics including electricity, magnetism, waves, and optics, this is the second course in a one-year sequence aimed at life sciences and premedical students. The two courses in this sequence are the largest offered by the physics department each year, with a typical enrolment of 180-220 students. A detailed list of topics can be found in the Appendix C.

The courses are well established and make extensive use of research-based pedagogical techniques in lectures, discussion sections, and weekly homework. The twice-perweek, 90-min-long lectures use an "interactive lecture" style involving small-group work interspersed with instructor feedback [27-32] and are led by an instructor experienced with this type of active learning [31]. The weekly discussion sections contain applications of the topics covered in lectures and are taught in the same active learning style; they are one-hour long and viewed as an extension of lecture, albeit in a smaller setting of about 15-20 students. The weekly homeworks are designed to reflect the principles of deliberate practice and feature "subskills" practice along with detailed and immediate feedback [23,33-35]. Both courses are well received and consistently earn strong student evaluations. With enrollees with similar backgrounds and similar pre-post scores on the Force Concept Inventory (FCI) [20], these courses have been shown to be remarkably stable year to year $[31,33]$. "One-minute" videos were introduced in the first semester of this course sequence in five years prior, after which, unprompted, approximately $25 \%$ of the students gave positive mention of them (e.g., "I loved the one-minute videos...") in course evaluations. In the three semesters prior to the study, students in this second semester course were also given access to "one-minute" videos along with short text summaries that highlight key points from the previous lectures (see examples in Fig. 1, Fig. 4, and the Appendix A). These "one-minute" videos, each with a duration of 1-2 min, are available for all major concepts in the course and have been widely used by students. In fact, in the most recent iteration of the course, $85 \%$ of students reported using the one-minute videos at least once during the semester, and a large fraction of them reported using the videos once per week. This positive feedback prompted the design of this study.

Our study sought to measure the impact of postlecture one-minute videos and short text summaries on students' problem-solving skills, along with their ability to chunk and organize information into a useful mental framework. The experimental intervention was conducted in a single 65-min session (see Fig. 2) in week 9 of a 15-week semester. All students in the course were invited to take part in the study $(N=222)$. Those who responded $(n=148)$ attended the one-hour session before the regularly scheduled class time. Students were told to bring their computers or mobile phones and headphones, as they may be assigned to a condition in which they would need computer access. As this study involved classroom-based research using normal educational practices, it was exempt from Institutional Review Board oversight.

To assess how different types of summary materials impact student learning, three groups were created (Fig. 2). All three groups were given access to the same set of annotated lecture and discussion section notes for the topic being covered in the experiment; the three groups differed with the kind of additional study materials they received. Students in group A had access to the one-minute videos only (experimental 1), while students in group B had access 


\section{$\underline{\text { Nearsightedness }}$}

When people are nearsighted, they cannot see things unless they are close. Their far point is not at infinity; it is often much closer.

To correct this, a diverging lens is placed in front of a person's eye to create a virtual image of the object at their reduced far point. The lens equation in this case becomes:

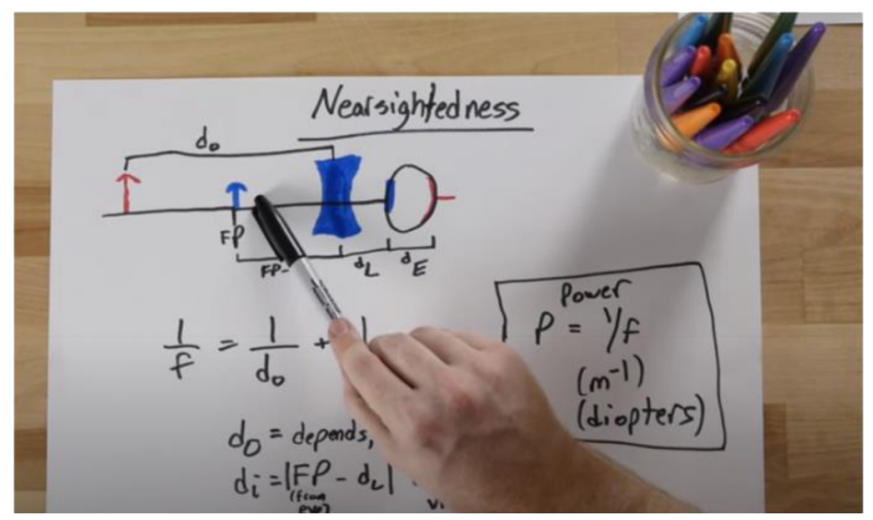

FIG. 1. Sample of integrated written and one-minute video summary materials for nearsightedness, one of the topics taught to students and explored in this study. The videos demonstrate different portions of the visual information to parallel the narration; they were created using simple handwritten materials and an overhead camera requiring less than 20 min per video to make, including planning, filming, and editing. A more complete example on Lenz's law (another topic in the course) can be found in Fig. 4 in Appendix A.

to the same one-minute videos embedded within short text summaries (experimental 2); see examples in Fig. 4 and the Appendix A. Students in group C were not given access to additional study materials (control) and studied using the annotated lecture and discussion section notes. It is important to note that the summaries provided to groups A and B did not contain new information that was otherwise missing from the annotated lecture and discussion section notes. With the goal of lowering students' cognitive load and help them organize the information, these short summaries described ideas and concepts succinctly with an emphasis on the links and connections between them. Common student difficulties and misconceptions were frequently noted and, when appropriate, contrasting cases were used to help deepen conceptual understanding and consolidation of information $[19,21,22,36]$. Groups were allocated based on the portion of the lecture hall they were seated in, side to

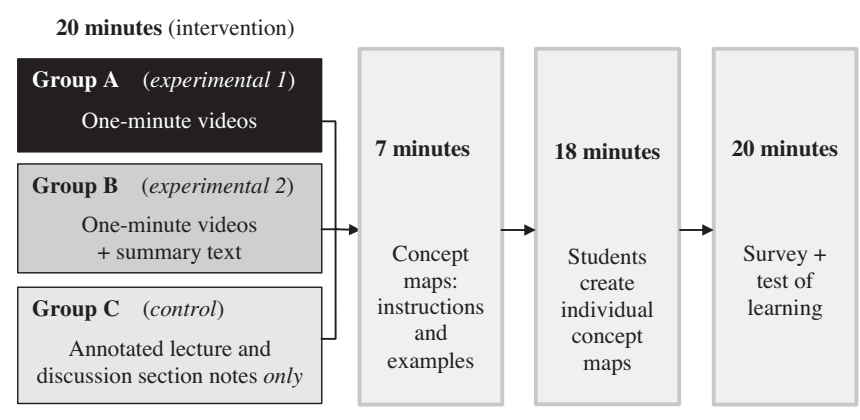

FIG. 2. Experimental design of the study. Students in all three groups were given 20 min to review their respective course materials for the module on thin optical lenses. Following a brief concept map tutorial $(7 \mathrm{~min})$, students were given $18 \mathrm{~min}$ to create a concept map for the thin optical lenses module. In the final $20 \mathrm{~min}$ of the session, students completed a brief survey and test of learning. side. While previous research indicates that there may be differences in student achievement as a function of distance to the front of the classroom [37], there is less concern for uneven distribution of students laterally. Nevertheless, the baseline differences across groups were assessed, and described below.

The study focused on a single module within the electricity and magnetism curriculum: thin optical lenses. This topic, which covers how thin lenses are used to focus light to create images and how lenses can be used for correcting abnormal eyesight, was selected because it is mostly self-contained compared to the other topics in the course. This selection allowed for a direct examination of learning as a function of the materials in question rather than a difference in knowledge between groups due to prior experience.

Following brief instructions, all students were provided handouts of the annotated lecture and discussion section notes for the thin optical lenses module. All students were given 20 min to study their respective course materials and then, at the end of this period, were asked to self-report the time-on-task along with their confidence in their level of preparedness for the course's weekly homework problems on a 5-point Likert scale (strongly agree, agree, neutral, disagree, strongly disagree). The next $45 \mathrm{~min}$ were used to evaluate students' conceptual understanding, problemsolving skills, and self-efficacy (see Fig. 2). The 45-min "evaluation period" consisted of three parts: (i) The first 7 min were used to tutor students on the making of a concept map, which included going over two examples of student-generated concept maps on Newton's 2nd law from another course. (ii) In the following $18 \mathrm{~min}$, students created a concept map for the content covered in the module. They were asked to place a particular emphasis on the connections between the various ideas and concepts 
TABLE I. Baseline characteristics by group. Groups do not appear to significantly differ after randomization across the first two exams in the course, suggesting that students in each group were similar. Because the exam scores are not normally distributed, the interquartile range (IQR) is used instead of standard deviation and $p$ is calculated using a Kruskal-Wallis test across groups.

\begin{tabular}{|c|c|c|c|c|}
\hline & Group A & Group B & Group C & \\
\hline & Videos & Videos + Summaries & & \\
\hline & (Experimental 1) & (Experimental 2) & (Control) & $p$ \\
\hline$n(\%$ total $)$ & $38(25.7 \%)$ & $71(48.0 \%)$ & $39(26.4 \%)$ & \\
\hline Midterm 1 Median (IQR) & $82.1(13.7)$ & $84.3(9.1)$ & $84.9(12.0)$ & 0.56 \\
\hline Midterm 2 Median (IQR) & $85.4(13.3)$ & $86.8(11.4)$ & $84.7(11.0)$ & 0.53 \\
\hline
\end{tabular}

(see the Appendix B). (iii) In the final phase of the evaluation period, students completed a 20-min test of learning (TOL) to assess their understanding of the content in the module. At the end of the 45-min evaluation period, the concept maps and TOL were collected. They were later evaluated by two blinded reviewers who scored the maps along the following three dimensions (1-3 points for each): content knowledge, relational knowledge, and clarity or accuracy. This allowed for a composite score (3-9) to be calculated. The TOLs were later evaluated by two blinded graders according to a standardized grading protocol, with partial credit awarded to students who made progress in solving questions but either did not finish or did not come to the correct final solution (0-30 points possible).

Baseline differences across groups $\mathrm{A}, \mathrm{B}$, and $\mathrm{C}$ were assessed using nonparametric $\chi^{2}$ and Kruskal-Wallis tests. Representativeness of the sample was also assessed by comparing participants to non-participants. All outcome analyses were conducted using non-parametric tests due to violations of parametric assumptions in the outcome data. Kruskal-Wallis tests were conducted to assess mediandifferences across TOL and concept map scores, along with self-reported confidence in completing homework assignments. When significantly different medians were detected across groups, tests of differences in the distributions of the outcomes were conducted using pairwise KolmogorovSmirnov tests. Despite nonparametric analyses, for visual representation of results, means and standard errors are used in accordance with typical visualization methods.

\section{RESULTS}

Out of 222 students, $148(66.7 \%)$ participated in the study. Groups (A: $n=38, \mathrm{~B}: n=71$, and C: $n=39$ ) were assigned based on lateral seating in the lecture hall, with the largest section of the seats corresponding to the middle group. The groups did not significantly differ by scores on the first midterm $(p=0.56)$ or second midterm $(p=0.53)$, suggesting that groups were balanced on students' prior knowledge and ability (Table I). Furthermore, we assessed the distribution of graduation years across groups, and found similar proportions of students in each year across the groups $(p=0.50)$.
The impact of the intervention was assessed with the following three measures:

(1) Concept map scores: Students in group A and students in group B scored similarly, with a median of 8.25 and 8, respectively [Fig. 3(a)]. These groups scored significantly higher than group $\mathrm{C}$, which had a median score of $6.5(p<0.001)$. To ensure that these scores were reliably graded, we estimated an intraclass correlation coefficient (ICC) based on a mean-rating, absolute agreement, two-way (i.e., two rater) random-effects model, consistent with our study design [38]. These findings suggest a strong reliability for the concept map scores $(\mathrm{ICC}=0.85$, $95 \%$ CI [0.80, 0.88], $p<0.001)$.

(2) TOL: Both types of summary materials significantly improve student understanding [Fig. 3(b)]. For the TOL, students viewing one-minute videos had a median score of 20.75 while students viewing oneminute videos and text summaries had a median score of 23 , both of which are significantly higher than students in group $\mathrm{C}$ that scored a median of

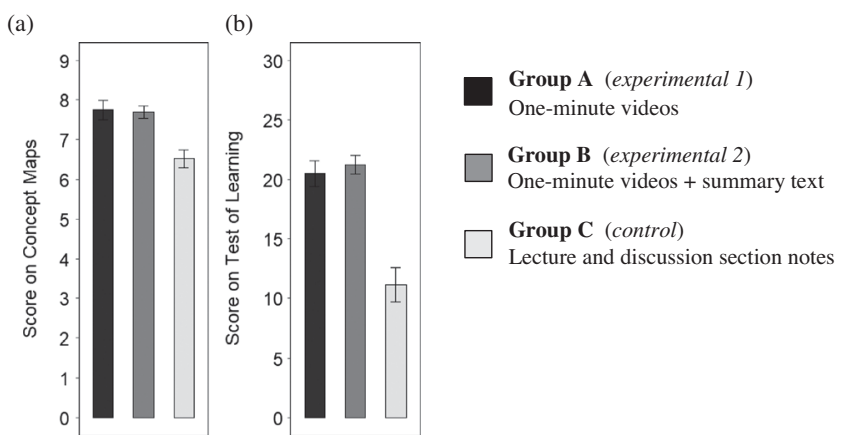

FIG. 3. Student performances on (a) concept maps and (b) test of learning. Scores on both types of assessments differed significantly across experimental and control groups $(p<0.001)$. These data suggest that groups with summary materials (groups A and B) had significantly better understanding than students who only had access to lecture materials without summaries (group C). Significance testing was done using Kruskal-Wallis tests (tests of medians). Bar height represents mean scores by group, and error bars represent \pm 1 standard error. 
$9(p<0.001)$. Considering that the concept map task took place before the test of learning, there is concern that the concept map activity may have influenced student performances on the TOL, and possibly provide additional benefits to the experimental condition. However, after adjusting for concept map scores using a linear regression model, students in the experimental groups (groups A and B) still scored significantly higher than students in the control group (group C), suggesting an independent effect of the materials themselves $(p<0.001)$. Furthermore, there is a significant moderate correlation between concept map scores and the TOL (Spearman $\rho=0.48$ ), indicating that the concept map scores constitute both a valid yet distinct measure of students' understanding. Taken in tandem, summary materials, as text or video, appear to significantly improve students' understanding across two valid, reliable measures.

Comparing the effectiveness of one-minute summary videos alone (group A) to one-minute summary videos with accompanying text (group B), there appeared to be no significant difference in distribution of TOL scores $(p=0.25)$ or concept map scores ( $p=0.51)$ across the two experimental groups; this suggests no significant difference in having oneminute videos compared to having one-minute videos and text. In total, these results strongly suggest that summary materials can improve student understanding of complex physics material in a controlled but realistic classroom setting.

(3) Homework self-efficacy: Following the concept map exercise, students used a 5-point Likert scale to evaluate the statement "After reviewing the material today, I feel confident that I can complete the homework." Experimental groups A and B reported increased confidence for completing the homework when compared to the control group $\mathrm{C}$ $(p<0.001)$, suggesting that carefully designed multimedia summaries may also increase student motivation $[8,17,24,39]$. Unfortunately, the homework scores cannot be used as a measure of learning for the following two reasons: first, all course materials, including the short multimedia summaries, were released to all students in the course at the end of the study, therefore allowing students in group $\mathrm{C}$ to use the short summaries before (and during) their homework. Second, since students are encouraged to work in groups during homework and are allowed multiple attempts for each problem (after receiving feedback), the homework grades are all saturated near $100 \%$ and cannot be used as a measure of learning for this reason. For ethical reasons, we did not include a question from module 7 on the final examination, which prevented it from being used as a measure of learning for this intervention.
On a survey given immediately following the study period, all 109 (100\%) students in the experimental groups $\mathrm{A}$ and $\mathrm{B}$ reported utilizing the summary materials during the allotted study time. More specifically, 81 (74.3\%) students in the experimental groups A and B exclusively used the written and video summary materials available. For the remaining $28(25.6 \%)$ students that still utilized lecture and discussion section materials, only $3(2.7 \%$ of total) used these materials for more than half of their allotted study time. These data suggest two key points. First, students in the experimental groups actually used the additional materials they were provided, as intended by the study protocols. Second, students overwhelmingly preferred the summary materials over fully annotated lecture and discussion section notes. However, the students that utilized any lecture and discussion section materials $(n=28)$ did not significantly differ from students that exclusively used summary materials $(n=81)$ on the test of learning $(p=0.17)$, concept map $(p=0.46)$, or selfreported confidence to complete the homework $(p=0.74)$.

We assessed the external validity of the sample by comparing students participating in the study $(n=148)$ and non-participants $(n=74)$; no meaningful differences were found between the groups on the module's homework grades $(p=0.19)$, number of attempts on the homework $(p=0.12)$ and scores on the course's three-hour, cumulative final exam $(p=0.08)$. Therefore, the participants in the study appear to be representative of the overall class population.

\section{DISCUSSION AND CONCLUSION}

Our study shows that carefully designed postlecture oneminute videos and short text summaries can be used to improve students' problem-solving skills as well as their ability to chunk and organize information into a useful mental framework. It is noteworthy that these learning improvements can be achieved at no appreciable cost to students' overall time on task. From the literature, we identify three likely factors responsible for these findings: (i) Chunking and organization of information can reduce students' cognitive load $[4,11]$; (ii) The use of contrasting cases can help students consolidate information and apply it to new contexts $[21,22,36]$; (iii) The crucial perspective of an expert tutor can ensure summaries address students' common difficulties and misconceptions at a level appropriate to their current level of understanding $[23,25,26,33,40]$. Below we describe some evidence suggesting that all three factors are involved and provide a detailed list of recommendations to help instructors develop effective multimedia summaries in their own courses.

The first factor that could account for these observations is the decrease in the extraneous cognitive load that students may experience during the learning process as a result of these short summaries [4,6,9-11]. By helping students organize a large number of concepts, think 
critically about the relationship between these concepts, and provide a framework by which to approach problems, these carefully designed summaries can result in cognitive processing that is more productive, thereby improving the germane cognitive load.

The summary materials were designed to be used soon after lecture; at that point in the learning cycle, students are likely to feel most overwhelmed with information because they are still processing novel information and incorporating it into a useful mental framework. To test these assumptions, we surveyed the next cohort of students $(n=215)$ on how (and when) they used the short summaries during the semester. The majority of students $(86 \%)$ reported using them at least once during the term, and while they had access to the summary materials at the beginning of each module, the majority of these students (94.8\%) chose to use the short summaries in the time interval between the end of lecture and the start of homework, with very few of them reporting using the summaries before lecture (i.e., pre-readings) or during (and after) homework. Their choice to use the short summaries soon after lecture is consistent with their intended use and indicates a critical need for additional resources at that point in the learning cycle. In fact, in response to the statement "I typically find myself most overwhelmed with new concepts and ideas," $1 \%$ responded "right after pre-reading," $74 \%$ "right after lecture," 20\% "when first starting the homework," none responded "right after finishing the homework," and 5\% responded "I never feel overwhelmed with the material." Their preference for using these short summaries at a time when they report feeling most overwhelmed with new concepts and ideas supports the idea that these types of summaries may help students process and consolidate the novel information, and in so doing, limit additional extraneous cognitive load while maximizing germane cognitive load.

A second contributing factor may be the short summaries' frequent use of contrasting cases designed to help students consolidate knowledge and improve subsequent problem solving. Prior research has demonstrated that seeing contrasting cases may help students learn novel physics material, particularly in active learning settings $[19,21,22,36,41,42]$. For example, when introducing the concept of net electric field from discrete charge distributions, the corresponding short summaries emphasized the similarities and differences in calculations as a function of symmetry in the charge distribution. Although the summary content is not designed to provide novel information beyond lectures, the contrasting cases used in this study may have helped students consolidate knowledge and enabled transfer to new tasks on the test of learning and in the concept map exercises.

Third, the short multimedia summaries may be most effective if they can accurately capture the student experience and conceptual frameworks for approaching the material $[23,25,26,40]$. With years of expertise and knowledge of more advanced topics, it is often difficult for advanced graduate students and faculty members to recognize and identify relevant aspects of student thinking. This common phenomenon, often referred to as expert blindness, can inhibit effective pedagogy $[43,44]$. On the other hand, a former student who recently learned the course material or a highly experienced teaching assistant in the course are both uniquely positioned to understand student thinking, and with additional training, may also be able to develop course materials that are well-matched to students' level of understanding [23,25,26,45-47]. In an undergraduate quantum mechanics course that was taught by one us, the short summaries developed in the early portion of the course received mixed reviews from students. This was in large part due to the fact that the instructor did not have sufficient knowledge of students' common difficulties and misconceptions and was therefore unable to produce short summaries that adequately targeted their thinking; prior research has demonstrated the importance of representing these misconceptions to maximize learning [48]. This was later addressed by hiring a former student who had done well in the course the previous year, and most importantly, had also interacted extensively with peers and developed an in-depth understanding of their thinking. For example, this student successfully identified the need to chunk the concept of complex functions down further into complex numbers in Cartesian and polar forms. This input enabled the instructor to later create one-minute videos and short summaries that were better targeted to student thinking; the feedback from students was immediate and overwhelmingly positive, with one student commenting that the one-minute videos "used to be dry and 'textbookish' [sic] but now they're full of info that's useful, and I feel much better prepared to work on my homework."

The success of this approach is consistent with research on the effectiveness of expert tutors $[26,40,49]$ and emphasizes the importance of instructors learning from effective tutoring practices, some of which include: having strong content knowledge; connecting to students' prior thinking and moving them into a more expert-like direction while providing them with timely and targeted feedback; maintaining a personal connection with students and motivating them to work hard by making the subject interesting and personally relevant. While the short summaries in this study are not (yet) interactive, they embody many of these same principles. A qualified former student or teaching assistant who both possess enough content expertise and extensive knowledge of how students think about the material can enable instructors to produce course content that is consistent with the most effective tutoring practices $[45-47,49]$.

These three factors are not entirely distinct and likely work in tandem. For instance, the effectiveness of the first 
TABLE II. A list of practical recommendations for effective summaries based on this study and the literature. The input from an expert tutor is critical in optimizing the effective of the summaries.

\begin{tabular}{|c|c|c|}
\hline $\begin{array}{l}\text { Characteristics of effective } \\
\text { short summaries }\end{array}$ & Description & References \\
\hline Focused on chunking & $\begin{array}{l}\text { Provide materials that break down the content into core concepts and ideas. The } \\
\text { connections between ideas should be made explicit. }\end{array}$ & {$[5,50,51]$} \\
\hline Timely deployment & $\begin{array}{l}\text { Summaries should be designed for use at the time when students are most } \\
\text { overwhelmed by content (i.e., after lecture) }\end{array}$ & {$[11,52]$} \\
\hline Short in length & $\begin{array}{l}\text { The goal of these short summaries is to help students synthesize the information } \\
\text { with no appreciable cost to the time on task. }\end{array}$ & {$[51,53,54]$} \\
\hline Incorporate student thinking & $\begin{array}{l}\text { Consider the approach students may take, which often differs from the approach } \\
\text { an expert may take. This may involve getting input from qualified former } \\
\text { students or highly experienced teaching assistants }\end{array}$ & {$[23,26,45-47]$} \\
\hline $\begin{array}{l}\text { Reduce unnecessary auditory } \\
\text { and visual stimulation }\end{array}$ & $\begin{array}{l}\text { Avoid too many bells and whistles; all materials in the summaries should help } \\
\text { student learning, not act as a distraction. Often, less is more. }\end{array}$ & {$[13,55,56]$} \\
\hline Identify useful cases & $\begin{array}{l}\text { Useful contrasting cases that illustrate important points can help students } \\
\text { integrate their ideas into useful schema. }\end{array}$ & {$[19,36,40]$} \\
\hline
\end{tabular}

two factors [i.e., (i) chunking and organizing; (ii) contrasting cases] are likely strongly dependent on each other. In turn, the most useful contrasting cases can impact students' ability to chunk and organize information, and yet, this ability to chunk and organize information is also likely to impact the effectiveness of the contrasting cases themselves-at minimum, it makes students better prepared to learn from them. This is why we view the development and use of contrasting cases as an integral part of the design for these short summaries. We strongly caution against viewing contrasting cases as something to be added later-on to improve already existing short summaries. On the one hand, a discipline expert (i.e., instructor) can find effective ways to help students chunk and organize information, including finding useful contrasting cases, and yet, for optimal impact, their effectiveness strongly depend on having the crucial input of an expert tutor $[40,43,45,49]$.

These three factors provide supportive evidence and explanations for the effectiveness of these carefully designed short summaries. In the following section, we translate these into actionable changes for instructors in their own courses. Based on these theories and literature across disciplines, we identified six recommendations (Table II). Along with these recommendations, we acknowledge that there may be financial or time constraints on developing these materials. We found that the creation of the summary video and written materials took the undergraduate teaching assistant less than $5 \mathrm{~h}$ each week. We believe this is attainable for many instructors, particularly since these materials can be recycled for further use in later semesters.

In conclusion, we find that the use of carefully designed one-minute videos and short text summaries following a lecture can significantly increase students' understanding of the material as well as their ability to organize the information into useful mental frameworks. These improvements can be achieved with a negligible increase in the overall time students spend on the material each week. These results, along with students' self-reports of feeling most overwhelmed "right after lecture," point to the critical need for additional resources at that point in the learning cycle that help students chunk and organize the information and reduce extraneous cognitive load during subsequent learning tasks. These short summaries have recently been deployed in several courses at Harvard University and the University of California Merced, and have enjoyed an overwhelmingly positive reception by students and instructors. In particular, engineering students in two different courses at UC Merced have also reported feeling overwhelmed "right after lecture." Notably, since all courses involved are using robust active learning strategies in lecture, in contrast to the majority of university courses in North America who still use a purely didactic lecture format [57], it is possible that the latter courses will enjoy a greater benefit from these carefully designed short multimedia summaries. In future work, we are investigating the impact of these short multimedia summaries on homework, exams, and long-term retention. The idea of chunking and organizing concepts before homework is of particular interest, in that this cognitive scaffolding may result in more productive time on task during homework. The cognitive principles involved in this study are well established and these results are likely to generalize across STEM disciplines.

\section{ACKNOWLEDGMENTS}

The authors are pleased to acknowledge significant contributions from Eric Mazur, along with valuable discussions with Melissa Franklin, Barak Gabai, Anna Klales, and David Morin. This work was supported by Harvard University through the Division of Science in the Faculty of Arts and Sciences. 
(a)

\section{Lenz's Law}

While Faraday's Law tells you the strength of the current induced, it doesn't tell you the direction of the current. Here comes Emil Lenz to the rescue.

This involves four steps:

1. Choose your area vector to point in the same direction as the original magnetic field. This ensures that your flux will be positive.

2. As you manipulate your system (via pulling the magnet, rotating things, etc.), is the flux through the loop increasing or decreasing?

3. If the flux is increasing, your loop will produce a magnetic field itself to counteract the increase; the induce magnetic field of the loop will point opposite to the increasing magnetic flux. If the flux is decreasing, your loop will produce a magnetic field in the same direction as the decreasing flux (to counteract this change). Note that step 3 is really what Lenz's law says.

4. Once you know your direction for which way your induced magnetic field from your loop will be, point your right thumb in that direction. Curl your fingers, and that's the direction the induced current will be!

(b)

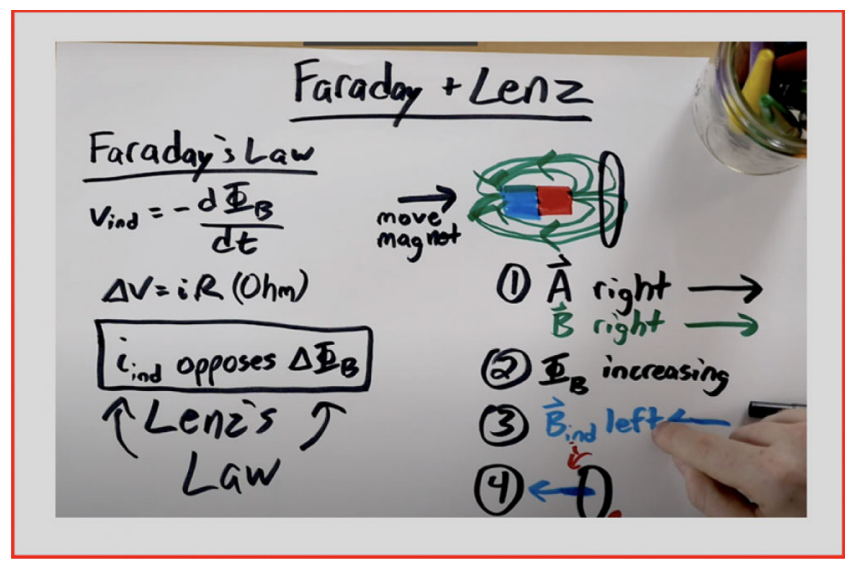

FIG. 4. (a) Short textual summary on the topic of Lenz's law. (b) Thumbnail of the one-minute video on Lenz's law. Further description is available in Appendix A.

\section{APPENDIX A: EXAMPLE OF SHORT MULTIMEDIA SUMMARY ON THE TOPIC OF LENZ'S LAW}

Figure 4 is an example of a multimedia summary that combines a one-minute video with a short text summary (i.e., group B: experimental condition 2.) In addition to highlighting key features of Lenz's law, this short summary chunks information into digestible steps that rely on different underlying concepts, including area vectors, magnetic flux, field induction, and the relationship between induced current and magnetic fields. The summaries are kept brief with a time on task of 1-3 min. For instance, the "one-minute" video that describes all of the important relationships between Faraday's and Lenz's laws has a time duration of 2 mins 27 secs [58]. The one-minute video ${ }^{1}$ contains highly effective contrasting cases, such as four different configurations for the relative orientation of the magnet and loop. This allows students to deduce deeper features of the physical system that are otherwise difficult to grasp.

\footnotetext{
${ }^{1}$ The one-minute video is embedded within the short text summary (thumbnail shown at the bottom).
} 


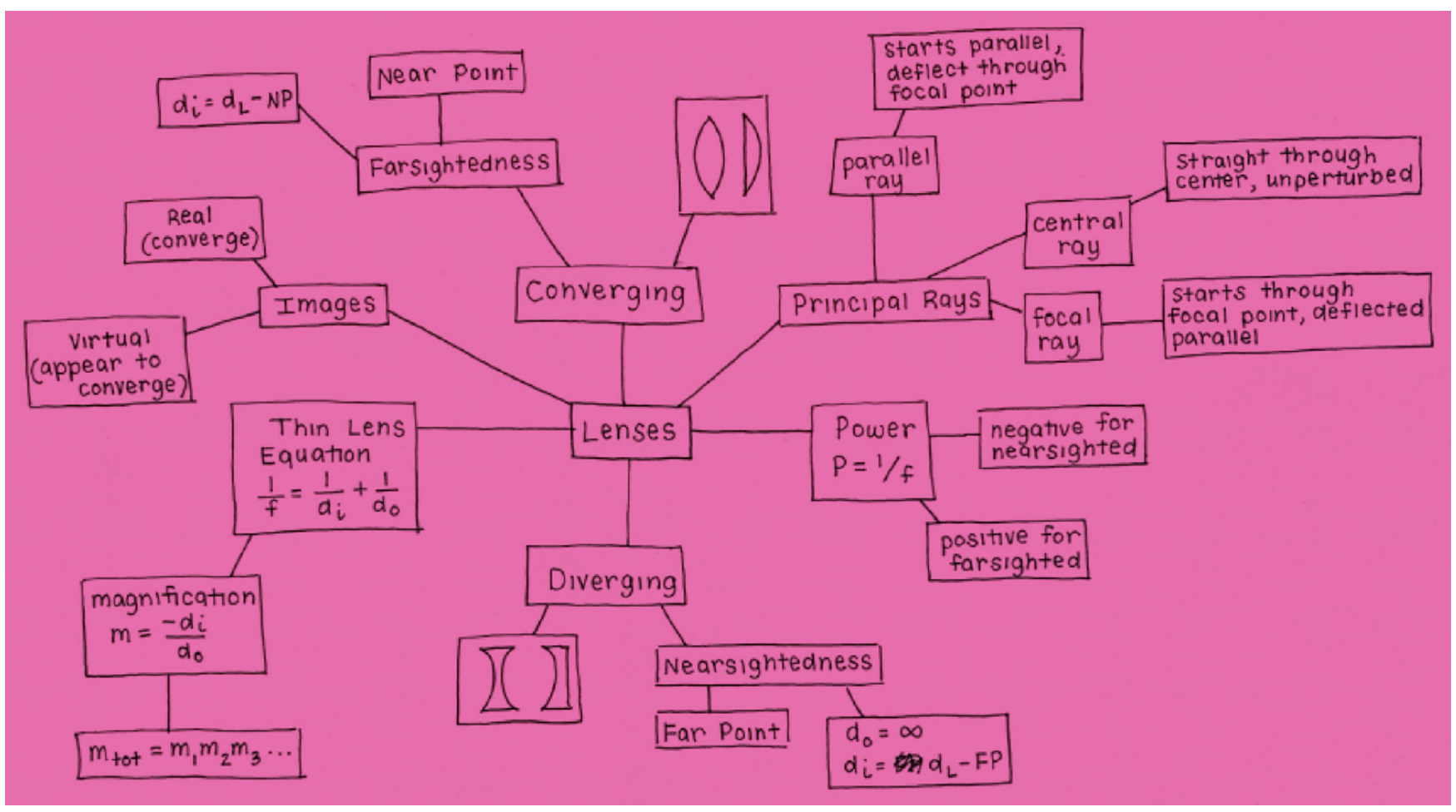

FIG. 5. Example of a strong concept map for assessing students' understanding of thin lenses and vision.

\section{APPENDIX B: EXAMPLE OF CONCEPT MAP COMPLETED BY A STUDENT IN THIS STUDY}

Figure 5 is an example of a concept map for assessing students' understanding of thin lenses and vision. This particular concept map received a high score; the connections between topics demonstrates a clear understanding of the material by the student. For example, this student demonstrates understanding of the connections between the type of lens and how it can be used to correct vision by linking diverging lens to near-sightedness and farsightedness to converging lens. Further, the student demonstrates understanding of the relationships for magnification of multiple lens systems by showing the total magnification as it relates to the definition of magnification for a single thin lens.

\section{APPENDIX C: LIST OF TOPICS COVERED IN THE ENTIRE COURSE FOR THIS STUDY}

The following shows a detailed list of topics for the course involved in this study: electricity, magnetism, waves, and optics. For each module that lasts 1 week, there are anywhere between 7 to 10 important subtopics (i.e., implicit chunking), with each subtopic having their corresponding short multimedia summary made of a short text and one-minute video.

TABLE III. Detailed list of topics in the course used for this study: Electricity, magnetism, waves, and optics.

\begin{tabular}{lcc}
\hline \hline Electric Potential and Coulomb's Law & Digital Circuits and Neurons & Light Rays and Reflection \\
Potential & Binary & Photons \\
Gradient & Digital logic & Electromagnetic waves \\
Force and gradient & Transistors & Refraction \\
Relating electrical quantities & Nernst and GHK & Mirror diagrams \\
Point charges and potential & Action potential & Mirror equation \\
Continuous charges and potential & Capacitors & \\
Coulomb's law & Potential propagation & Lenses, Refraction, and Vision \\
Conductivity and resistivity & & Diverging lenses \\
& & Converging lenses
\end{tabular}


TABLE III. (Continued)

Electric Potential and Coulomb's Law

Current, Resistance, and Capacitance

Intro to capacitors

Capacitors and circuits

Resistors and circuits

Navigating circuits

Electrical power

Kirchoff's junction rule

Kirchoff's loop rule

Electric Fields and Gauss's Law

Electric fields

Electric dipoles

Dielectrics

Electric flux

Gauss's law
Digital Circuits and Neurons

Magnetism, Spin, and Angular Momentum

Magnetic fields

Wire magnetic fields

Lorentz forces

Magnetic dipoles

Right Hand Rules

Magnetic Spin

MRI Overview

MRI Magnetization

MRI-Rotating the M Vector

MRI Gradients

Flux and Faraday

Faraday and Lenz
Light Rays and Reflection

Lens equation

Combining optical elements

Biology of vision

Nearsightedness

Farsightedness

Waves, Interference, and Diffraction Intensity

Wave interference

Wave reflections

Interference-two slits

Single slit diffraction

Interference and diffraction Airy disks

Thin media interference

Microscopy and Sound

Microscopes

Numerical aperture

Harmonics

Doppler effect

Polarization and malus
[1] N. Cowan, Z. Chen, and J. N. Rouder, Constant capacity in an immediate serial-recall task: A logical sequel to Miller (1956), Psychol. Sci. 15, 634 (2004).

[2] A. Baddeley, The magical number seven: Still magic after all these years?, Psychol. Rev. 101, 353 (1994).

[3] G. A. Miller, The magical number seven, plus or minus two: Some limits on our capacity for processing information, Psychol. Rev. 63, 81 (1956).

[4] I. Koch, A. M. Philipp, and M. Gade, Chunking in task sequences modulates task inhibition, Psychol. Sci. 17, 346 (2006).

[5] B. B. De Koning, H. K. Tabbers, R. M. J. P. Rikers, and F. Paas, Towards a framework for attention cueing in instructional animations: Guidelines for research and design, Educ. Psychol. Rev. 21, 113 (2009).

[6] J. Sweller, Cognitive load theory, learning difficulty, and instructional design, Learn. Instr. 4, 295 (1994).

[7] J. J. G. Van Merriënboer and J. Sweller, Cognitive load theory and complex learning: Recent developments and future directions, Educ. Psychol. Rev. 17, 147 (2005).

[8] R. E. Mayer, The promise of multimedia learning: Using the same instructional design methods across different media, Learn. Instr. 13, 125 (2003).

[9] T. de Jong, Cognitive load theory, educational research, and instructional design: Some food for thought, Instr. Sci. 38, 105 (2010).
[10] F. Paas and T. van Gog, Optimising worked example instruction: Different ways to increase germane cognitive load, Learn. Instr. 16, 87 (2006).

[11] F. Gobet, Chunking models of expertise: Implications for education, Appl. Cogn. Psychol. 19, 183 (2005).

[12] C. Leopold, E. Sumfleth, and D. Leutner, Learning with summaries: Effects of representation mode and type of learning activity on comprehension and transfer, Learn. Instr. 27, 40 (2013).

[13] R. E. Mayer, W. Bove, A. Bryman, R. Mars, and L. Tapangco, When less is more: Meaningful learning from visual and verbal summaries of science textbook lessons, J. Educ. Psychol. 88, 64 (1996).

[14] M. Schneider and F. Preckel, Variables associated with achievement in higher education: A systematic review of meta-analyses, Psychol. Bull. 143, 565 (2017).

[15] S. Elena Gallagher, M. O’Dulain, N. O'Mahony, C. Kehoe, F. McCarthy, and G. Morgan, Instructor-provided summary infographics to support online learning, EMI Educ. Media Int. 54, 129 (2017).

[16] A. King, Comparison of self-questioning, summarizing, and notetaking-review as strategies for learning from lectures, Am. Educ. Res. J. 29, 303 (1992).

[17] L. Fiorella and R. E. Mayer, Eight ways to promote generative learning, Educ. Psychol. Rev. 28, 717 (2016).

[18] T. Stelzer, G. Gladding, J. P. Mestre, and D. T. Brookes, Comparing the efficacy of multimedia modules with 
traditional textbooks for learning introductory physics content, Am. J. Phys. 77, 184 (2009).

[19] G. Kestin, K. Miller, L. S. McCarty, K. Callaghan, and L. Deslauriers, Comparing the effectiveness of online versus live lecture demonstrations, Phys. Rev. Phys. Educ. Res. 16, 013101 (2020).

[20] D. Hestenes, M. Wells, and G. Swackhamer, Force Concept Inventory, Phys. Teach. 30, 141 (1992).

[21] W. A. Wickelgren, Chunking and consolidation: A theoretical synthesis of semantic networks, configuring in conditioning, S-R versus cognitive learning, normal forgetting, the amnesic syndrome, and the hippocampal arousal system, Psychol. Rev. 86, 44 (1979).

[22] D. L. Schwartz, C. C. Chase, M. A. Oppezzo, and D. B. Chin, Practicing versus inventing with contrasting cases: the effects of telling first on learning and transfer, J. Educ. Psychol. 103, 759 (2011).

[23] C. Wieman, Applying new research to improve science education, Issues Sci. Technol. 29, 25 (2012), https://www .jstor.org/stable/43315691.

[24] A. Bandura and E. A. Locke, Negative self-efficacy and goal effects revisited, J. Appl. Psych. 88, 87 (2003).

[25] C. E. Wieman, Expertise in University Teaching \& the implications for teachin effectiveness, evaluation \& training, Daedalus (Boston) 148, 47 (2019).

[26] W. B. Wood and K. D. Tanner, The role of the lecturer as tutor: Doing what effective tutors do in a large lecture class, CBE Life Sci. Educ. 11, 3 (2012).

[27] L. Deslauriers, E. Schelew, and C. Wieman, Improved learning in a large-enrollment physics class, Science 332, 862 (2011).

[28] L. Deslauriers and C. Wieman, Learning and retention of quantum concepts with different teaching methods, Phys. Rev. ST Phys. Educ. Res. 7, 010101 (2011).

[29] J. D. Jones, W. K. Madison, and C. Wieman, Transforming a fourth-year modern optics course using a deliberate practice framework, Phys. Rev. ST Phys. Educ. Res. 11, 020108 (2015).

[30] L. Deslauriers, L. S. McCarty, K. Miller, K. Callaghan, and G. Kestin, Measuring actual learning versus feeling of learning in response to being actively engaged in the classroom, Proc. Natl. Acad. Sci. U.S.A. 116, 19251 (2019).

[31] L. S. McCarty and L. Deslauriers, Transforming a large university physics course to student-centered learning, without sacrificing content, The Routledge International Handbook of Student-Centered Learning and Teaching in Higher Education (Routledge, London, 2020) pp 186-200.

[32] J. Beyer, H. Strobelt, M. Oppermann, L. Deslauriers, and H. Pfister, Teaching visualization for large and diverse classes on campus and online, in Proceedings of IEEE VIS Workshop on Pedagogy Data Visualization (IEEE, Bellingham, WA, 2016).

[33] K. Miller, K. Callaghan, L. S. McCarty, and L. Deslauriers, Increasing the effectiveness of active learning using deliberate practice: A homework transformation, Phys. Rev. Phys. Educ. Res. 17, 010129 (2021).

[34] K. A. Ericsson, Acquisition and maintenance of medical expertise: A perspective from the expert-performance approach with deliberate practice, Acad. Med. 90, 1471 (2015).

[35] K. A. Ericsson, R. Krampe, and C. Tesch-Romer, The role of deliberate practice in the acquisition of expert performance, Psychol. Rev. 100, 363 (1993).

[36] E. Kuo and C.E. Wieman, Toward instructional design principles: Inducing Faraday's law with contrasting cases, Phys. Rev. Phys. Educ. Res. 12, 010128 (2016).

[37] V. Tagliacolloab, G. Volpatoac, and A. Junior, Association of student position in classroom and school performance, Educ. Res. 1, 198 (2010).

[38] T.K. Koo and M. Y. Li, A guideline of selecting and reporting intraclass correlation coefficients for reliability research, J. Chiropr. Med. 15, 155 (2016).

[39] M. A. Rittmayer and M. E. Beier, Self-Efficacy in STEM, in Applying Research to Practice (ARP) Resources, edited by B. Bogue and E. Cady (2009). Retrieved from http:// aweonline.org/arp_selfefficacy_overview_122208.pdf.

[40] K. A. Ericsson, Deliberate practice and acquisition of expert performance: A general overview, Acad. Emerg. Med. 15, 988 (2008).

[41] J. L. Quilici and R.E. Mayer, Role of examples in how students learn to categorize statistics word problems, J. Educ. Psychol. 88, 144 (1996).

[42] B. Rittle-Johnson and J. R. Star, The power of comparison in learning and instruction: Learning outcomes supported by different types of comparisons, Psychol. Learn. Motiv. Cogn. Educ. (Elsevier Academic Press, San Diego, CA, US, 2011), pp. 199-225.

[43] M. Nathan, K. Koedinger, and M. Alibali, Expert blind spot: When content knowledge eclipses pedagogical content knowledge, in Proceedings of the Third International Conference on Cognitive Science (2001).

[44] M. J. Nathan and A. Petrosino, Expert blind spot among preservice teachers, Am. Educ. Res. J. 40, 905 (2003).

[45] V. Otero, S. Pollock, and N. Finkelstein, A physics department's role in preparing physics teachers: The Colorado Learning Assistant Model, Am. J. Phys. 78, 1218 (2010).

[46] L. S. Shulman, Those who understand: Knowledge growth in teaching, Educ. Res. 15, 4 (1986).

[47] A. Maries and C. Singh, Exploring one aspect of pedagogical content knowledge of teaching assistants using the test of understanding graphs in kinematics, Phys. Rev. Phys. Educ. Res. 9, 020120 (2013).

[48] D. A. Muller, J. Bewes, M. D. Sharma, and P. Reimann, Saying the wrong thing: Improving learning with multimedia by including misconceptions, J. Comput. Assist. Learn. 24, 144 (2008).

[49] M. R. Lepper and M. Woolverton, The wisdom of practice: Lessons learned from the study of highly effective tutors, in Improving Academic Achievement, edited by J. Aronson (Academic Press, New York, 2002), pp. 135-158.

[50] R. E. Mayer and R. Moreno, Nine ways to reduce cognitive load in multimedia learning, Educ. Psychol. 38, 43 (2003).

[51] M. Ibrahim, P. D. Antonenko, C. M. Greenwood, and D. Wheeler, Effects of segmenting, signalling, and weeding on learning from educational video, Learn. Media Technol. 37, 220 (2012). 
[52] F. E. Ritter, J. Nerb, E. Lehtinen, and T. M. O'Shea, Order to Learn: How the Sequence of Topics Influences Learning (Oxford University Press, Oxford, England, 2007).

[53] D. Zhang, L. Zhou, R. O. Briggs, and J. F. Nunamaker, Instructional video in E-Learning: Assessing the impact of interactive video on learning effectiveness, Inf. Manag. 43, 15 (2006).

[54] G. D. Rey, M. Beege, S. Nebel, M. Wirzberger, T. H. Schmitt, and S. Schneider, A meta-analysis of the segmenting effect, Educ. Psychol. Rev. 31, 389 (2019).
[55] S. Kalyuga, P. Chandler, and J. Sweller, Managing splitattention and redundancy in multimedia instruction, Appl. Cogn. Psychol. 13, 351 (1999).

[56] M. Fyfield, M. Henderson, and M. Phillips, 25 principles for effective instructional video design, in Proceedings of the Australasian Society for Computers in Learning in Tertiary Education (Singapore University of Social Sciences, Singapore, 2019), pp. 418-423.

[57] M. Stains et al., Anatomy of STEM teaching in North American universities, Science 359, 1468 (2018).

[58] https://www.youtube.com/watch?v=CwI8ZG43rTg. 